\title{
Politique
}

Politique

\section{La politique d'internationalisation de Montréal : une politique en devenir}

\section{Micheline Plasse}

Numéro 19, hiver 1991

URI : https://id.erudit.org/iderudit/040681ar

DOI : https://doi.org/10.7202/040681ar

Aller au sommaire du numéro

Éditeur(s)

Société québécoise de science politique

ISSN

0711-608X (imprimé)

1918-6584 (numérique)

Découvrir la revue

Citer cet article

Plasse, M. (1991). La politique d'internationalisation de Montréal : une politique en devenir. Politique, (19), 37-65. https://doi.org/10.7202/040681ar
Résumé de l'article

Cet article porte sur la question de l'« internationalité » des grandes villes et plus spécifiquement de l'« internationalité » de Montréal. Partant d'une démarche qui repose à la fois sur l'étude des déclarations publiques et des stratégies mises de l'avant par les acteurs institutionnels, l'article veut faire état des réalisations, des contraintes et des perspectives d'avenir que suscite cette question.

Montréal compte plusieurs avantages favorisant son internationalisation, bien que plusieurs facteurs nuisent à ce développement, notamment cette difficulté qu'ont les acteurs à se concerter sur le devenir de cette métropole. Les données recueillies lors de cette étude montrent toutefois qu'une dynamique nouvelle émerge entre les acteurs à l'effet de remplacer l'attentisme du passé face aux gouvernements supérieurs par une prise en charge qui devrait permettre de développer une vision à long terme. En revanche, nous ne pouvons pas encore parler d'une véritable politique d'internationalisation de la ville de Montréal mais d'une politique d'internationalisation encore en devenir. 


\section{La politique \\ d'internationalisation \\ de Montréal' :}

une politique en devenir

Micheline Plasse

Université du Québec à Montréal

Cet article porte sur la question de l' «internationalité» des grandes villes et plus spécifiquement de I' «internationalité" de Montréal. Partant d'une démarche qui repose à la fois sur l'étude des déclarations publiques et des stratégies mises de l'avant par les acteurs institutionnels, l'article veut faire état des réalisations, des contraintes et des perspectives d'avenir que suscite cette question.

Montréal compte plusieurs avantages favorisant son internationalisation, bien que plusieurs facteurs nuisent à ce développement, notamment cette difficulté qu'ont les acteurs à se concerter sur le devenir de cette métropole. Les données recueillies lors de cette étude montrent toutefois qu'une dynamique nouvelle émerge entre les acteurs à l'effet de remplacer l'attentisme du passé face aux gouvernements supérieurs par une prise en charge qui devrait permettre de développer une vision à long terme. En revanche, nous ne pouvons pas encore parler d'une véritable politique d'internationalisation de la ville de Montréal mais d'une politique d'internationalisation encore en devenir.

Depuis quelques années, l'internationalité des grandes villes est une nouvelle problématique dans l'étude des relations et des acteurs internationaux'. Ainsi, il ne se passe pas une semaine sans qu'il ne soit question, dans les diffé-

* Le terme «Montréal» englobe ici Montréal et sa grande région.

1. Pour un relevé de la littérature sur le rôle international des cités, voir P. Soldatos «Atlanta and Boston in the New International Cities Era : Does Age Matter?» dans E. Fry, L.M. Radebaugh et P. Soldatos, The New International Cities Era, Provo, Utah, Brigham Young University, 1989, pp. 37-72. Voir également P. Soldatos, Les nouvelles villes internationales : profil et planification stratégique, Édition Serdeco, Aix-En-Provence, 1991. 
rents médias, du statut ou du caractère international de Montréal. Plusieurs facteurs socio-économiques et politiques militent en faveur du développement du rôle international des grandes villes. Parmi les plus importants, citons : la libéralisation des échanges et des investissements étrangers; la passation aux gouvernements régionaux et locaux ${ }^{2}$ des responsabilités des gouvernements supérieurs aux prises avec des problèmes budgétaires; l'émergence de pays nouvellement industrialisés; l'expansion sans précédent du commerce mondial; l'intérêt accru des citoyens pour les problèmes internationaux. L'exemple des villes américaines témoigne de cet état de fait. En effet, plusieurs d'entre elles ont fortement accru leur présence dans le champ des relations internationales au cours des dernières années ${ }^{3}$.

Les villes canadiennes n'ont pas échappé à ce courant. Ainsi, les villes de Montréal, d'Ottawa et de Toronto se sont dotées, ou sont en voie de le faire, de structures administratives dont relèvent les questions à caractère international. Le présent article porte exclusivement sur le cas de Montréal et veut faire état des réalisations, des contraintes et des perspectives que suscite le développement de mesures visant à accentuer le caractère international de la métropole du Québec.

Notre démarche repose sur l'étude des déclarations publiques et des stratégies mises de l'avant par les acteurs institutionnels et également sur une enquête menée auprès de ceux-ci. Plus précisément, les résultats examinés ici proviennent de deux sources principales. Nous avons d'abord

2. Pour une réflexion sur la crise au niveau local, voir M. Gottliener, The Decline of Urban Politics, Political Theory and the Crisis of the Local State, Beverly Hills, Sage Publications, 1987. Dans ce contexte, pour une réflexion sur l'évolution du fédéralisme, voir John Kincaid, «Implications of Constituant Diplomacy for the European Communities», Paper prepared for the Annual Conference of the International Association of Centers for Federal Studies and the Comparative Federalism and Federation Research Committee, Bruges, College of Europe, Groupe Condenbergh, 26-29 octobre 1989.

3. Heidi H. Hobbs, American Cities in International Perspective : Local Governments in Foreigh Affairs, thèse de doctorat non publiée, University of Southern California, décembre 1989, p. 2. 
analysé les rapports Picard ${ }^{4}$ et L'Allier ${ }^{5}$, documents qui ont grandement contribué à l'émergence d'une pensée un peu plus articulée sur la question. Une étude documentaire et des entrevues ${ }^{6}$ complètent l'analyse.

Les entrevues ont été réalisées auprès d'acteurs privilégiés, actifs ou intéressés par cette nouvelle réalité : MM. Jean-Paul L'Allier et Laurent Picard, M. Jacques Ménard, exprésident de la Chambre de commerce de Montréal, ainsi que d'une dizaine de fonctionnaires et de hauts fonctionnaires des divers paliers gouvernementaux (fédéral, provincial, Communauté urbaine de Montréal et ville de Montréal). Le questionnaire soumis à ces témoins et à ces acteurs privilégiés portait principalement sur la perception de leur rôle, sur leurs attentes, sur les actions qu'ils préconisaient pour favoriser le développement du caractère international de Montréal et sur les problèmes et contraintes qu'ils rencontraient dans la mise en œuvre de ces actions.

Avant de définir le cadre d'analyse et de faire état des résultats de notre étude, il convient d'énumérer brièvement les critères de l'internationalité d'une grande ville. Qu'est-ce qu'une ville internationale? En partant d'un idéal-typique et selon Panayotis Soldatos, une ville internationale peut être définie comme la somme et l'interpénétration de plusieurs caractéristiques. La quantification de ces critères, bien que complexe, constitue le cœur de la définition d'une "ville internationale ${ }^{7}$ ". Ainsi, selon Panayotis Soldatos, directeur

4. Canada, Rapport du Comité consultatif au Comité interministériel sur le développement de la Région de Montréal, Ottawa, ministère des Approvisionnements et Services du Canada, novembre 1986.

5. Jean-Paul L'Allier et Associés Inc., Montréal pour une stratégie d'intervention en matière de relations internationales, Montréal, Étude réalisée sur mandat du Comité exécutif de la ville de Montréal, le 25 novembre 1987.

6. Ces entrevues ont été réalisées entre les mois de septembre 1989 et juillet 1990.

7. P. Soldatos, "Atlanta and Boston...", op. cit., p. 38, propos traduits ici par l'auteure de cet article. 
général de l'Institut d'étude des villes internationales, les principaux critères d'internationalité d'une ville sont les suivants:

- avoir une situation géographique qui lui donne une visibilité internationale;

- bénéficier de facteurs de production étrangers (capital, main-d'œuvre et services) et être engagée dans diverses transactions économiques;

- être le siège d'institutions étrangères et internationales;

- accueillir sur son territoire des entreprises et des institutions économiques installées aussi à l'étranger;

- avoir des moyens de transport directs et efficaces avec l'étranger;

- être engagée dans des activités de communication avec des pays étrangers (tourisme, échange d'étudiants, missions de commerce, etc.) et être dotée d'un important réseau de télécommunications;

- disposer d'équipements suffisants pour accueillir des activités à caractère international (centre de congrès, salles d'exposition, hôtels, centres de recherche, etc.);

- avoir à sa disposition des médias jouissant d'une audience et d'un rayonnement à l'échelle internationale;

- être régulièrement l'hôte d'événements prestigieux (expositions, festivals, événements sportifs, etc.);

- posséder des institutions nationales, régionales ou même locales ayant une réputation et un rayonnement internationaux lassociations, universités, centres de recherche, musées, etc.);

- être dotée d'institutions publiques et privées qui sont parties à des ententes de collaboration avec l'étranger (dans les domaines social, culturel ou économique);

- pouvoir compter sur un appareil administratif pour conduire de façon systématique une "paradiplomatie»;

- avoir une population composée de diverses nationalités ${ }^{8}$.

8. Ibid., p. 39. 
Mais comme le souligne le professeur Soldatos, le profil international d'une ville ne peut pas être tributaire de ces seuls traits situationnels. II faut, précise encore cet auteur, que les acteurs veuillent "d'abord penser international et [qu'ils] recherchent par des actions l'internationalisation ${ }^{9}$ ".

Montréal possède plusieurs de ces avantages. Sans reprendre systématiquement tous les critères mentionnés cidessus, Anne Francis et Pierre-Paul Proulx ont montré que Montréal peut être une "cité internationale dynamique» si tous les niveaux de gouvernement, de concert avec le secteur privé, décidaient de collaborer. Pour ces auteurs, les forces de Montréal résident principalement dans la stabilité de son climat économique et social et dans sa qualité de vie. De plus, l'aide que les gouvernements apportent à la région pour attirer les industries, les liens que possède la région en matière de télécommunication, les ententes de coopération avec différents partenaires ainsi que la présence de diplomaties locales accentuent ce caractère international. Enfin, la présence de représentants étrangers, de populations étrangères et la tenue d'événements présentés par d'autres pays constituent aussi des facteurs à ne pas sous-estimer. En revanche, Anne Francis et Pierre-Paul Proulx déplorent certaines faiblesses dans la structure industrielle de Montréal, dans ses liens commerciaux et dans son activité en bourse. Ils soulignent aussi le petit nombre de sièges sociaux et l'insuffisance des équipements nécessaires aux milieux $d^{\prime}$ affaires internationaux ${ }^{10}$.

9. Panayotis Soldatos, «Les contours essentiels de la nouvelle génération de villes internationales : causalité du phénomène, profil international et stratégies de développement", texte de synthèse élaboré pour le Colloque de la TRIADE (Amérique-Europe-Asie), «Les journées de Strasbourg : séminaire franco-américain» des 8 et 9 décembre 1988, (texte dactylographié).

10. Anne Francis et Pierre-Paul Proulx, «A Comparison of Selected Indicators of International and Economic Activities in Montréal and New Yorkw, dans E. Fry, L.H. Radebaugh et P. Soldatos, The New International Cities Era, op. cit., p. 157. 


\section{Le cadre d'analyse}

Selon Ivo D. Duchacek", il y a deux façons pour un sous-groupe national ou pour un acteur infra-étatique de participer délibérément aux relations internationales. L'une est de tenter d'influencer le processus décisionnel du gouvernement central en faisant du lobbying autant au niveau exécutif qu'au niveau législatif. L'autre est de contourner les gouvernements supérieurs en maintenant des contacts formels ou informels avec des voisins ou des sources de pouvoir.

Les gouvernements régionaux et municipaux qui choisissent la seconde voie s'exposent à agir dans un contexte de compétition directe, voire de conflit avec les gouvernements supérieurs. Par ailleurs, les grandes villes représentent des sites vitaux pour la santé économique et sociale d'un État. Nous reprenons ici l'idée émise par Jane Jacobs, selon laquelle l'intérêt pour les questions internationales dans une ville "constitue une façon plus pertinente et plus précise de travailler à la croissance économique d'un pays ${ }^{12}$ ". Dans ce contexte, comment les villes peuvent-elles influencer les processus décisionnels des gouvernements supérieurs?

Pour saisir l'idée que se font de la question les acteurs institutionnels montréalais et leurs vis-à-vis des gouvernements supérieurs, nous avons choisi l'approche de Pierre Muller et Bruno Jobert. Cette approche allie développement des politiques et réflexion sur la place de l'État dans les sociétés modernes. Le schéma que proposaient les deux

11. Ivo D. Duchacek, «Multicommunal and Bicommunal Politics and Their International Relations», dans Ivo D. Duchacek, Daniel Latouche et Gaith Stevenson, Perforated Sovereignties and International Relations : Transsovereign Contacts of Subnational Governments, New York, Greenwood Press, 1989, pp. 3-28. Voir aussi du même auteur *The International Dimensions of Self government , Publius : The Journal of Federalism, $n^{\circ} 14$, automne 1984, pp. 5-31.

12. Jane Jacobs, Cities and the Wealth of Nations, New York, Random House, 1984, cité dans A. Francis et P.-P. Proulx, "A Comparison of Selected...», op. cit., p. 120. 
auteurs porte sur les politiques sectorielles définies comme une intervention publique basée sur deux logiques : d'abord, la logique territoriale et, ensuite, la logique sectorielle. Ainsi, les politiques d'aménagement du territoire ou de décentralisation appartiennent-elles à la première catégorie alors que les politiques de santé ou de transport, pour ne citer que ces exemples, relèvent de la seconde ${ }^{13}$.

Dans ce cadre, "le référentiel sectoriel est donc un construit social. C'est une image qui est elle-même le produit des rapports de force dans le secteur. Souvent, la structure même du référentiel reflètera un compromis entre les différentes élites en compétition au sein du secteur ${ }^{14} "$. Les identités professionnelles se substituent aux identités locales. $\mathrm{Ce}$ processus de sectorisation crée de nouveaux modèles d'intervention étatique qui vont donner naissance à des politiques sectorielles, elles-mêmes constitutives de l'État moderne.

Toujours selon Muller et Jobert, nous assisterions donc au passage de la territorialité à la sectorialité comme mode d'organisation dominant de la société, et "chaque ensemble sectoriel issu du processus de sectorisation va développer une logique de reproduction autonome ${ }^{15}$ ". Pour Muller, cette société sectorisée a nécessairement besoin d'une instance extérieure pour réguler sa propre reproduction. Cette instance, c'est l'État, qui utilise les "politiques sectorielles» comme moyens d'action. Dans une telle optique, l'objet de ces

13. Pierre Muller, «Un schéma d'analyse des politiques sectorielles», Revue française de science politique, vol. 35, $n^{\circ} 2$, avril 1985, p. 168.

14. Bruno Jobert et Pierre Muller, L'État en action - politiques publiques et corporatismes, Paris, Presses universitaires de France, 1987, p. 69. Selon ces auteurs, une politique publique se présente toujours sous deux aspects principaux : d'abord une ximage sociale, c'est-à-dire une représentation du système sur lequel on veut intervenir"; ensuite «un ensemble de moyens organisationnels, financiers, administratifs, juridiques, humains, bref des procédures, des techniques, des relations de pouvoir, tout ce qui fait qu'une politique $n^{\prime}$ est pas seulement un processus intellectuel mais un processus social concret* (p. 51).

15. Pierre Muller, "Un schéma...", loc. cit., p. 168. 
politiques consiste à maintenir ou à restaurer au sein de la "sectorialité" un minimum de cohérence, lequel était autrefois assuré par la territorialité.

La sectorialisation des sociétés entraîne deux conséquences importantes : apparition de ce que Muller considère comme des crises de régulation; émergence de problèmes liés aux corporatismes sectoriels ${ }^{16}$. Ainsi, les politiques publiques expriment l'exigence de "régulation» des sociétés modernes. Dans ce contexte, une théorie des politiques publiques doit donc nécessairement s'intégrer dans une théorie de l'État. Ce dernier devient ainsi le lien de «régulation" générale d'où émergent de multiples politiques.

Muller termine son article en disant que nous assistons aujourd'hui à un retour au "territorial». II y voit une tentative de désectoriser l'action de l'État en intégrant les différents pôles d'intervention : "on découvre aujourd'hui qu'une société ne peut pas complètement abandonner son principe de territorialité ${ }^{17}$. Dans ce contexte, nous serions en "panne de référentiel», et il y aurait crise des projets modernisateurs en raison de la montée des corporatismes sectoriels (agricoles, médicaux, routiers, etc.), montée qui ne serait que l'expression de l'impossibilité pour une société sectorielle de se passer de référentiel. En somme, nous assistons à une double crise : panne de croissance économique et panne de référentiel idéologique.

Selon Jobert et Muller, une politique sectorielle doit nécessairement prendre en compte trois éléments fondamentaux qui sont autant de clefs pour comprendre selon quelle logique fonctionne l'intervention de l'État dans tel ou tel domaine :

- Une politique sectorielle, c'est d'abord une tentative pour gérer un rapport entre le secteur concerné et la société globale : c'est le rapport global-sectoriel;

16. Les groupes sociaux tendent à transformer leurs objectifs sectoriels en fins ultimes, c'est-à-dire en intérêt général.

17. Ibid., p. 186. 
- Ce rapport global-sectoriel ne se transforme en un objet d'intervention publique qu'en fonction de l'image que s'en font les acteurs concernés. On appellera cette représentation référentiel terme qui désigne l'ensemble des normes ou des référents d'une politique. La question qui se pose alors est de déterminer comment est construit ce référentiel qui est en quelque sorte l'acte constitutif de toute politique;

- Enfin, il reste à savoir qui se charge de cette opération de construction de référentiel. En d'autres termes, quel est l'acteur qui va réellement élaborer l'image du système à réguler? À partir de quels concepts se fera cette élaboration? En même temps, il faut s'interroger sur le róle joué par les acteurs dans l'établissement de nouveaux rapports de pouvoir dans le secteur ${ }^{18}$.

La politique à l'étude ici est une politique plus multisectorielle que sectorielle; son but est d'intégrer la dimension territoriale. Dans ce contexte, les deux dernières questions citées ci-dessus se posent davantage que la première et serviront de guide à notre analyse. Donc, quelle image se font les acteurs de l'intervention publique en matière d'internationalisation de Montréal, quels sont les acteurs les plus actifs et leur rôle dans l'élaboration de cette politique?

\section{La ville de Montréal et la Communauté urbaine de Montréal : un leadership certain}

II ne fait pas de doute que les représentants de la ville de Montréal ont une volonté politique d'affirmer et de développer le caractère international de celle-ci. Le maire Jean Doré l'exprimait en ces termes le 22 novembre 1988 :

18. Bruno Jobert et Pierre Muller, «L'État en...», op. cit., p. 52. 
Nous avons pris I'habitude, chez nous, de confondre les événements internationaux ponctuels et le caractère international permanent d'une ville. Les grands événements internationaux qui se sont déroulés à Montréal depuis vingt ans ont eu un impact et un retentissement considérables. II faut maintenant aller plus loin et se donner une stratégie de développement qui vise autant à améliorer nos propres avantages comparatifs qu'à faire mieux connaître Montréal aux quatre coins du monde ${ }^{19}$.

Le maire ne faisait ici que réitérer ce qu'il avait déjà transmis à sa machine bureaucratique. En effet, le premier fonctionnaire de la ville, M. Pierre Lefrançois, disait en mars 1987 : "La ville est très consciente de la dimension concurrentielle des grandes métropoles entre elles[...]. C'est ainsi que l'on mène présentement divers travaux permettant $\mathrm{d}^{\prime}$ améliorer le positionnement international de Montréal ${ }^{20}$ ". L'analyse de l'ensemble de ces discours montre clairement que l'administration montréalaise recherche la collaboration active de ses partenaires.

Dans cet esprit et à la suite d'une recommandation du Rapport L'Allier', la Ville de Montréal mettait sur pied un secrétariat aux relations internationales. Ce service devait permettre à Montréal de ne plus être à la remorque des gouvernements supérieurs tout en lui donnant la possibilité de

19. Jean Doré, Allocution prononcée devant le Conseil des relations internationales de Montréal, 22 novembre 1988, polycopié, pp. 2-3.

20. Pierre Lefrançois, «Un plan de travail pour I'administration montréalaise», Notes du secrétaire général de la Ville de Montréal, conférence prononcée dans le cadre des dîners-conférences organisés par l'Institut d'administration publique du Canada, Groupe régional de Montréal, 10 mars 1987, pp. 7 et 12 .

21. Jean-Paul L'Allier et Associés Inc., «Montréal pour une...», op. cit. 
définir ses propres règles de fonctionnement sur la scène internationale.

Jusqu'à maintenant, le secrétariat a concentré son action sur les dossiers suivants : maintien de la communication avec les 42 consulats ayant pignon sur rue à Montréal; organisation des missions du maire à l'étranger; rétablissement des contacts avec I'Organisation de l'aviation civile internationale (OACI); (seul organisme des Nations Unies présent à Montréal, il menaçait de déplacer ses quartiers généraux à Genève); signature d'ententes de coopération avec la ville d'Abidjan; obtention du sommet des 25 plus grandes villes du monde en 1991. Le secrétariat travaille également sur des dossiers ponctuels majeurs, tel celui de la création du Centre des conférences internationales.

De toute évidence, la création du secrétariat a amélioré les relations entre les édiles municipaux et les organisations étrangères présentes sur son territoire. Ainsi $M$. Drogini $M$. Fiorita, alors directeur de la délégation canadienne à l'OACl, disait en août 1989 au journaliste Gérald Leblanc : "Depuis douze mois, il y a eu une amélioration de $500 \mathrm{p}$. cent dans nos relations avec la Ville. C'est le jour et la nuit, depuis la création du Secrétariat aux affaires internationales. Maintenant, nous avons un interlocuteur à l'Hôtel de ville ${ }^{22}$ ".

La Ville a aussi adopté d'autres mesures pour améliorer ses avantages comparatifs et resserrer les liens entre les divers intervenants internationaux en place à Montréal. Ce sont la nomination d'une conseillère au poste de responsable politique des dossiers internationaux; la création au service de la planification et de la concertation d'une unité chargée d'établir des liens avec les chercheurs universitaires qui travaillent en collaboration avec des pays étrangers; la consolidation du mandat de la Commission d'initiative et de développement économique de Montréal (CIDEM); l'octroi d'un mandat analogue à la Commission d'initiative et de développement culturel (CIDEC) en ce qui a trait aux activités internationales de leur clientèle; le resserrement de la collaboration entre la

22. Gérald Leblanc, «Drogini Fiorita», La Presse, 2 août 1989, p. B-3. 
Ville et l'Office d'expansion économique de la CUM $^{23}$ responsable de la prospection d'investissements étrangers.

Tous nos interlocuteurs s'entendent pour dire que la ville de Montréal tient un discours cohérent, que le maire a pris le leadership dans plusieurs dossiers et que la Ville a pris les mesures «pour faire face à son rôle international».

En revanche, la CUM n'a pas encore de vision articulée du rôle qu'elle pourrait exercer dans le développement d'activités internationales dans la région de Montréal. Pour le moment, les dossiers se traitent à la pièce. La CUM a toutefois commencé à établir des paramètres d'intervention et a fait avancer la question en proposant la création d'une table de concertation qui regroupe les principaux acteurs montréalais. Initiative qui aura sûrement des effets positifs sur l'accentuation du caractère international de Montréal ${ }^{24}$.

23. La Communauté urbaine de Montréal a été mise sur pied en 1969 et constitue une forme de gouvernement régional responsable de grands dossiers tels ceux de la police, de la protection de l'environnement, de l'alimentation en eau, ainsi que certains dossiers liés à la culture; l'abréviation CUM désignera dorénavant cette organisation.

24. Ce Comité de concertation économique du territoire de la CUM s'est donné un mandat qui s'articule autour de cinq points : se concerter sur des enjeux majeurs, définir des orientations communes, développer une vision globale du développement de Montréal et de sa région, chercher l'adhésion sur cette vision globale du développement et harmoniser les actions des nombreux acteurs. Ce comité est formé de décideurs représentant les organismes suivants : la Communauté urbaine de Montréal (le président, l'adjoint au président et un conseiller); la ville de Montréal (le viceprésident du comité exécutif de la ville et le directeur du CIDEM); la Chambre de commerce du Montréal métropolitain (son président et son vice-président); le Board of Trade of Montreal (le président et le vice-président); des représentants des syndicats (le président du Conseil central de Montréal de la Confédération des syndicats nationaux et le président du Conseil des travailleurs et travailleuses du Montréal métropolitain affilié à la Fédération des travailleurs et travailleuses du Québec). Les gouvernements fédéral et provincial sont d'accord pour participer aux travaux de ce comité sur une base ad hoc, à titre de participants-invités intéressés à des sujets spécifiques. 


\section{Les gouvernements fédéral et provincial : des acteurs de soutien}

Jusqu'à présent, les gouvernements supérieurs $n$ 'ont pas été indifférents au dossier du développement international de Montréal. Cependant, ils ont agi à la pièce sans avoir de véritable stratégie d'ensemble. Ainsi le gouvernement québécois prend parti pour sa métropole. Citant l'ancien ministre des Relations internationales, le sous-ministre de ce même ministère, dans une allocution prononcée le 22 novembre 1988, appuyait le développement international de Montréal $^{25}$. Malheureusement, ce discours se contentait de souligner les projets ou les affaires déjà en cours et $n$ 'annoncait aucune nouvelle initiative venant concrétiser ses propos. Néanmoins, un peu plus tard, le gouvernement a approuvé trois mesures importantes qui pourraient contribuer à transformer le discours en actions.

En juin 1989, le gouvernement du Québec a adopté une réglementation accordant des immunités et des privilèges fiscaux aux fonctionnaires d'organismes internationau $x^{28}$. Cette mesure annoncée dans le discours du budget de 19891990 devait pouvoir favoriser l'implantation d'organismes internationaux au Québec. Ainsi, des organismes internationaux reconnus, gouvernementaux ou non gouvernementaux, peuvent bénéficier de privilèges fiscaux : exemption d'impôt sur le revenu des particuliers, exemption de taxes à la consommation, exemption de certaines taxes municipales et scolaires autres que celles perçues sur les services.

Dans ce même esprit, le gouvernement québécois adoptait en juin 1989 des législations pour modifier les chartes de

25. Allocution de $M$. Jean-Claude Villard, sous-ministre des Affaires internationales du gouvernement du Québec, prononcée le 22 novembre 1988 au Colloque sur «Montréal, ville internationale», document polycopié, p. 3.

26. Le Règlement sur le régime fiscal municipal et scolaire applicable aux gouvernements des autres provinces, aux gouvernements étrangers et organismes internationaux, 1989, 121, G.0., II, 5256. 
Montréal et de Québec de façon à étendre leur marge de manœuvre en matière de négociation d'ententes avec des organismes étrangers ${ }^{27}$.

Enfin, réalisant une promesse électorale faite lors de la campagne de 1989, le gouvernement créait un comité ministériel permanent chargé du développement de la région de Montréal ${ }^{28}$. Ce comité a notamment pour mandat de mettre en place une structure de concertation entre les principaux acteurs associés au développement de Montréal. Selon les mots mêmes du Premier ministre, Robert Bourassa, ce comité devrait constituer «le principal levier de développement économique du Québec". Mieux que des promesses d'investissement de la part du gouvernement, cette initiative devrait confirmer le rôle de Montréal comme «ville du monde et ville d'avant garde» a encore fait valoir le Premier ministre ${ }^{29}$.

27. Loi modifiant la charte de la Ville de Montréal, Gouvernement du Québec, 1989, c. 80. Cette loi a été sanctionnée le 22 juin 1989.

28. Ce comité se compose des représentants des ministères suivants : le président du Conseil du Trésor qui préside le comité, la vice-première ministre et ministre de l'Énergie et des Ressources, le ministre de la Maind'œuvre et de la Sécurité du Revenu, le ministre des Transports, le ministre de l'Industrie, de la Science et de la Technologie, le ministre du Tourisme, la ministre des Communications et le ministre des Communautés culturelles. M. Ronald Poupart, conseiller du Premier ministre, qui a le statut d'un secrétaire général associé, assure la permanence. M. Poupart peut compter sur les services d'une dizaine de personnes pour le seconder dans ses tâches.

29. Bernard Descôteaux, «Montréal jouira d'un statut particulier», Le Devoir, 12 septembre 1989, p. 2. Le comité a tenu deux rencontres avec les représentants du milieu montréalais siégeant pour le Comité de concertation économique du territoire de la CUM, la première en février 1990 et la seconde le 15 juin 1990. Le comité peut saisir le Conseil des ministres soit de sa propre initiative, soit à la demande des principaux acteurs de Montréal. Fait à noter, le ministre des Affaires internationales ne siège pas à ce comité. Ce ministère a été créé en décembre 1988 afin d'unifier l'action du gouvernement du Québec à l'étranger. II concentre les activités des ministères des Relations internationales et du Commerce extérieur, exception faite des responsabilités du domaine de la technologie et de la prospection des investissements étrangers, qui sont confiées au ministère de l'Industrie du Commerce et de la Technologie comme sont aussi exclues les activités 
Par ailleurs, le gouvernement fédéral a participé activement à la discussion sur le développement international de Montréal notamment dans la foulée des recommandations du Rapport Picard, présenté en novembre 1986. Le comité de travail présidé par M. Laurent Picard, professeur à l'université McGill, devait soumettre au gouvernement fédéral des recommandations visant à assurer la rentabilisation de l'économie montréalaise. Ce comité devait aussi formuler un plan d'action pour mettre ces recommandations en œuvre.

Le Rapport Picard a d'abord posé un diagnostic et a bien montré que les gouvernements successifs, tant fédéral que provincial, ont largement négligé la région de Montréal. II a aussi démontré que ces gouvernements $n^{\prime}$ ont pas su coordonner leurs politiques et leurs choix de projets dans divers domaines. Le diagnostic pèse lourd : manque d'efforts concertés, absence de vision d'ensemble, carence quant à la définition d'objectifs, en somme absence d'unité de pensée et d'action. Le rapport déplore du même coup les batailles que se sont livrées les gouvernements entre eux ${ }^{30}$. En 1990, plusieurs de nos interlocuteurs ont formulé la même critique.

En réponse à cette situation, le Rapport Picard proposait plusieurs mesures pour actualiser la relance de l'économie de Montréal. Plusieurs d'entre elles devaient contribuer en même temps à faire de Montréal une véritable ville internationale. Le rapport proposait sept axes de développement permettant à la métropole de faire face à la concurrence internationale : 1. les activités internationales; 2 . la haute technologie; 3 . la finance et le commerce international; 4 . le design; 5 . les industries culturelles; 6 . le tourisme; 7 . le transport.

Au chapitre des stratégies mises de l'avant pour atteindre les objectifs de développement, le rapport prônait la coopération intergouvernementale, la coopération entre les universités et les entreprises, l'établissement de relations patronales-

canadiennes hors Québec, qui relèvent du Secrétariat aux Affaires intergouvernementales canadiennes du Conseil exécutif.

30. Rapport du Comité... op. cit., p. XIX. 
syndicales harmonieuses et l'adoption de politiques fiscales concurrentielles $^{31}$. En conclusion, le rapport demandait au secteur privé de prendre le leadership, et aux gouvernements de se concerter formellement pour contribuer à la réalisation $d^{\prime} u n$ vaste plan de développement ${ }^{32}$.

Le gouvernement fédéral a répondu en publiant une série d'engagements qui allaient dans le sens des recommandations du Rapport Picard ${ }^{33}$. Mais M. Picard estime que le gouvernement fédéral n'a pas tenu ses engagements. L'auteur n'a pas caché son amertume et a dénoncé à plusieurs reprises et sur plusieurs tribunes l'action du fédéral, allant même jusqu'à prétendre que son rapport a été appliqué à Toronto.

En entrevue, M. Picard cite des exemples et dit ne pas comprendre l'inaction du Gouvernement qui refuse toujours de signer la convention des Nations Unies sur les privilèges et les immunités. Cette signature donnerait aux organisations internationales les mêmes avantages fiscaux que ceux que leur a octroyés le gouvernement du Québec ${ }^{34}$.

Par ailleurs, nos autres interlocuteurs s'entendent pour dire que le gouvernement fédéral n'a pas véritablement favorisé Montréal en créant pour cette ville et pour celle de Vancouver des centres financiers internationaux qui sont, disent-ils, "des coquilles vides" : "On a eu peur de déplaire à l'Ouest du pays et à Toronto". Cette évaluation confirme l'opinion émise par le ministre de l'Industrie, des Sciences et de la Technologie, Benoît Bouchard, et selon laquelle «il est devenu difficile, à Ottawa, de faire avancer les dossiers montréalais ou québécois sans susciter de ressentiments». Son collègue, le

31. Ibid., p. 48.

32. Ibid., p. XXVI, pp. 165-166.

33. Canada, Pour le développement d'une métropole, Ottawa, ministère des Approvisionnements et Services, 1987.

34. Plusieurs des interlocuteurs rencontrés partagent l'opinion de $\mathbf{M}$. Picard sur cette question. Le gouvernement fédéral refuserait de ratifier cette convention pour des motifs d'équité fiscale envers tous les Canadiens. 
ministre fédéral du Travail, Jean Corbeil, rappelait à la Chambre des communes en février 1990 qu'il revient en premier aux intervenants montréalais d'assurer leur développement économique, que le gouvernement fédéral n'a pas à être le maître d'œuvre de la relance économique de Montréal : "Nous considérons notre intervention comme étant de faciliter les choses et non de les exécuter ${ }^{35}$ ". Un haut fonctionnaire fédéral nous confirme cette position :

Le fédéral fonctionne beaucoup à l'anglosaxonne, c'est-à-dire en traitant les dossiers au cas par cas, de façon ad hoc et sur la base de la collaboration des trois niveaux de gouvernement. Nous avons une approche de matching funds et notre politique en est une de ciblage mais sur la base du Rapport Picard. Mais, il reste que c'est délicat pour le fédéral d'avoir une politique de développement pour une ville en matière internationale. S'il fait quelque chose pour une ville, il devra aussi faire la même chose pour les autres villes.

Selon nos interlocuteurs fédéraux, la stratégie du gouvernement central s'appuie sur la philosophie du Rapport Picard. Le gouvernement doit "mettre l'accent sur des investissements stratégiques pour donner un coup de pouce mais sans prendre la place du secteur privé». Le gouvernement fédéral "est prêt à faire des choses mais il ne faudrait pas que les coûts soient trop élevés". Tous les fonctionnaires fédéraux se disent fort préoccupés du déficit du gouvernement canadien et soulignent l'étroite marge de manœuvre du gouvernement.

35. Marianne Favreau, «Ottawa épaulerait Montréal dans son projet de Centre de conférences», La Presse, 16 octobre 1989, p. A-3, et Jean Corbeil, «À Montréal d'agir d'abordw, La Presse, 3 février 1990, p. G-1. 
Selon cette perspective, le gouvernement fédéral a pris des mesures qui s'inscrivent dans la logique du Rapport Picard : adoption d'une législation sur les produits pharmaceutiques, création de l'Agence spatiale, attribution de subventions à des musées montréalais, octroi de sommes pour des études de préfaisabilité au sujet du Centre international de Montréal. Mais plusieurs de nos répondants insistent pour qu'au-delà de ces actions s'établisse une véritable coordination entre les trois ministres responsables du développement de la région de Montréal : Jean Corbeil pour Montréal, Monique Landry pour le nord de la région et Benoît Bouchard pour le sud de la région. Ils insistent aussi pour que la haute fonction publique, "centralisatrice par nature et par intérêt", accorde plus d'attention aux besoins de la région de Montréal.

De plus, les hauts fonctionnaires fédéraux affirment que le gouvernement fédéral n'a pas intérêt comme son vis-à-vis provincial à créer un comité ministériel : "Un tel comité susciterait les jalousies des autres provinces canadiennes et il faudrait en créer pour toutes les autres villes». Les propos des fonctionnaires fédéraux interviewés et les déclarations des politiciens montrent que le gouvernement fédéral considère que son rôle est de faciliter et d'appuyer tout essor économique et non d'en être le maître d'œuvre. Politiciens comme fonctionnaires précisent aussi que ces appuis devraient être apportés en tenant compte des contraintes budgétaires. Dans cet esprit, la nomination de $M$. JeanClaude Lebel, haut fonctionnaire de carrière, au poste de sousministre rattaché à Montréal augure bien pour la suite du dossier.

L'action engagée par les milieux montréalais semble confirmer l'idée émise par $M$. Jean-Paul L'Allier, alors aspirant à la mairie de Québec ${ }^{36}$, selon laquelle les gouvernements fédéral et provincial ne uferont jamais beaucoup parce que ces deux gouvernements sont obligés de faire des choix. Ils vont saupoudrer leurs efforts en tenant compte de faits internes

36. Propos recueillis en entrevue. 
alors qu'ils devraient tenir compte de faits liés au marché, et le gouvernement fédéral notamment ne fera jamais ces choix. II ne faut pas compter sur les gouvernements supérieurs; il faut plutôt s'en servir, car les gouvernements vont plier devant les plus forts». M. L'Allier ajoutait que le réseau diplomatique favorisera davantage Toronto que Montréal parce que c'est le réseau qu'il connaît le mieux.

Ce tableau de la situation resterait partiel sans l'analyse de la participation de plus en plus active de la Chambre de commerce du Montréal métropolitain.

\section{La Chambre de commerce de Montréal : un acteur-clé dans la nouvelle dynamique montréalaise}

Le Rapport Picard notait que le secteur privé de Montréal avait été "remarquablement passif» comparativement aux secteurs privés de Toronto et de Boston. Depuis quelques années, et plus particulièrement depuis la tenue du Sommet économique de la ville de Montréal en juin 1986, le milieu des affaires a joint sa voix à celle des nombreux intervenants qui manifestent une volonté et un enthousiasme certains face à la nécessité d'une coopération entre tous les acteurs.

Au cours des deux dernières années, les actions et prises de position de la Chambre de commerce du Montréal métropolitain ont grandement contribué à la création d'une nouvelle dynamique à l'intérieur de laquelle la Chambre a accordé une place privilégiée au développement d'activités internationales. Ainsi, en 1988, en collaboration avec le Bureau de commerce, la Chambre a fait un effort de réflexion et de concertation qui a débouché sur l'opération Option Montréal. De cette opération se dégagent trois consensus décisifs pour l'avenir de Montréal : le besoin d'une vision globale du développement de cette ville; la nécessité d'une concertation soutenue entre les différents acteurs; la volonté de prendre une part active au processus décisionnel. 
Dans ce cadre, la Chambre considère que «le développement international d'une métropole est une condition sine qua non de son développement interne ${ }^{37}$. Avec la participation des médias et de personnalités de divers milieux, la Chambre a donc organisé, en mars 1989 , une vaste campagne publicitaire sur le thème "Montréal oblige" afin de promouvoir une stratégie commune de développement économique.

Cette volonté de s'attaquer aux problèmes de fond du développement économique de Montréal s'est réaffirmée et s'est consolidée au cours du mandat de l'ex-président de la Chambre, $M$. Jacques Ménard ${ }^{38}$. Ce dernier a fortement insisté tout au long de la dernière année sur la nécessité de "travailler dans une optique de solidarité, de connivence et de complicité avec les différents paliers de gouvernement ${ }^{39}$ ». Cette réflexion sur le développement des activités internationales à Montréal s'inscrit elle aussi dans un cadre plus large, celui de la nécessaire coopération des acteurs :
II y a cinq ans, la Chambre de commerce avait un discours dépassé. Elle essaie à présent de présenter à ses membres les vrais enjeux, de leur donner une vision globale. Les gens d'affaires de la région se sont donné une structure pour se concer-

37. Chambre de commerce du Montréal métropolitain, «Les grandes questions d'aménagement dans la région de Montréal : Option Montréal, le point de vue du milieu des affaires», document polycopié, août 1988, p. 13. Voir aussi le texte d'une communication de Serge Godin, président de la Chambre de commerce de Montréal, au Colloque «Montréal, ville internationale?», le 22 novembre 1988, ENAP-GERFI, CORIM.

38. Signalons à cet égard que la Chambre a modifié son appellation de Chambre de Commerce de Montréal pour Chambre de commerce du Montréal métropolitain de manière à étendre son action et à intégrer des représentants de la Rive Sud et de la ville de Laval.

39. L. Jacques Ménard, président de la Chambre de commerce du Montréal métropolitain, "Ville en liberté surveillée de loin», La Presse, 9 février 1990, p. B-3. 
ter : le Conseil permanent d'initiatives du Montreal métropolitain (COPIMM) ${ }^{40}$ pour que les diverses Chambres de la région articulent des positions communes ${ }^{41}$.

La Chambre suit deux axes prioritaires : la concertation des gouvernements et l'établissement de consensus entre les gens d'affaires eux-mêmes : "Nous devons être plus cohérents face à des gouvernements qui ne pensent pas suffisamment Montréal| ${ }^{42}$.

\section{Éléments de discussion}

À la lumière des propos théoriques de Muller et de Jobert déjà cités, nous pouvons mieux comprendre la position des principaux acteurs institutionnels. La politique, ou plutôt l'embryon de politique, d'internationalisation de la ville de Montréal constitue, selon la théorie de Muller, un retour de la "territorialité" et une tentative de réunir les acteurs institutionnels les plus influents autour du développement de l'ensemble des politiques sectorielles.

Dans le cadre de la politique étudiée, nous pouvons dire que la Ville de Montréal, la CUM et la Chambre de commerce du Montréal métropolitain constituent les principaux acteurs sociaux qui ont pris le leadership de l'internationalisation de la région de Montréal, problématique qui s'inscrit dans le contexte plus large, du développement économique et social de la région métropolitaine.

40. Créé suite à l'opération Option Montréal, ce comité de concertation formé en 1989 regroupe des gens d'affaires, et doit promouvoir une dynamique régionale pour les dossiers ayant une incidence sur le rayonnement de la région de Montréal.

41. L. Jacques Ménard, président de la Chambre de commerce du Montréal métropolitain, «Ville en ...», loc. cit.

42. Propos recueillis en entrevue. 
Incidemment, ces acteurs partagent un certain nombre de points de vue et, ce faisant, contribuent à l'élaboration d'un "référentiel normatif» qui devrait servir de base à des actions concrètes permettant d'actualiser une véritable politique d'internationalisation de Montréal. Retenons ici les points de vue qui sont le plus souvent énoncés et que partage le plus grand nombre :

Premier consensus : il faut accentuer la coopération entre les différents acteurs afin de contribuer à la planification et à la réalisation d'un vaste plan de développement qui miserait sur les forces du milieu; les acteurs s'entendent pour que le développement d'activités qui accentueraient le caractère international de Montréal et de sa région fasse partie intrinsèque de ce vaste plan ${ }^{43}$.

Selon M. Picard, ces problèmes seront résolus en grande partie quand il y aura davantage d'entrepreneurs politiques, plus préoccupés de développement que de leur champ de juridiction : "Qu'ils agissent et s'ils débordent leur champ de juridiction, ils se le feront dire et iront devant les tribunaux ${ }^{44}$ ".

Second consensus : les gouvernements supérieurs portent un intérêt encore trop peu marqué au développement de la région de Montréal.

À cet égard, M. L'Allier n'est pas tendre envers les fonctionnaires provinciaux qu'il qualifie de rabat-joie, qui essaient de "tasser le maire pour passer le ministre, fonctionnaires qui, dit-il, se voient comme surveillants des gouverne-

43. Les mesures à prendre en matière de choix des équipements culturels constituent un exemple de ces déficiences : la saga de la construction d'une salle de concert pour l'Orchestre symphonique de Montréal qui s'affirme de plus en plus internationalement témoigne de ces imbroglios qui marquent l'action des gouvernements.

44. Propos recueillis en entrevue. 
ments municipaux»; "le gouvernement provincial a plutôt tendance à prendre ombrage du développement international de ses villes ${ }^{45}$ ".

Troisième consensus : on reconnaît l'importance des difficultés liées à l'existence d'une multitude de programmes gouvernementaux et au manque de communication entre les ministères, voire à l'existence de certaines rivalités bureaucratiques.

Plusieurs de nos interlocuteurs signalent également que ces difficultés peuvent être surmontées et citent en exemple la mise sur pied du Centre d'initiative de haute technologie, un dossier qui représente pour eux l'exemple-type d'une coopération fructueuse entre tous les acteurs.

Quatrième consensus : on note la propension du milieu montréalais à "faire du tapage» autour d'un dossier plutôt que de miser sur le plus long terme, une situation qu'alimenterait cette idée d'une province de Québec plus choyée que ses consœurs en matière d'attention fédérale.

Au Québec, nous disait M. L'Allier, «il y a un danger, c'est de prendre nos discours pour des réalités; avant de se donner des objectifs, il faudrait d'abord avoir une "hygiène urbaine", c'est-à-dire bien prendre soin de soi comme ville et bien prendre soin de nos représentants étrangers œuvrant dans cette ville ${ }^{46}$ ". Dans une certaine mesure, cette critique

45. M. L'Allier donne l'exemple du projet de l'ACDI (Agence canadienne de développement international), Afrique 2000, qui n'a aucunement profité aux villes du Québec en raison de l'inaction du gouvernement provincial.

46. Propos recueillis en entrevue. 
maintes fois émise explique la discrétion entourant les études de faisabilité d'un centre de conférences internationales ${ }^{47}$.

Par ailleurs, notre analyse documentaire et nos entrevues montrent que la politique d'internationalisation de Montréal en est davantage une d'adaptation au contenu du rapport socioéconomique qu'une politique qui anticipe sur l'évolution de ce rapport. En effet, les acteurs socio-économiques locaux ${ }^{48}$ ont pris les devants et ont en quelque sorte mis de l'avant, comme préalable à l'action, une stratégie de prise en charge et d'activisme. Dans une telle optique, les acteurs locaux en viennent à penser eux aussi que les gouvernements supérieurs doivent être des soutiens et non des initiateurs.

Mais les acteurs institutionnels locaux savent pertinemment qu'une ville ne peut relever seule le défi du développement $^{49}$ non plus que celui de l'internationalisation. Les gouvernements supérieurs doivent l'appuyer dans cette entreprise.

Dans cet esprit, il faudrait, dans un exercice ultérieur, confronter les intentions et les représentations des acteurs avec des critères de mesure comme ceux qu'a élaborés Panayotis Soldatos. Un tel exercice permettrait d'évaluer dans quelle mesure Montréal s'internationalise ou pas et aiderait à départager entre ce qui tient du mythe et ce qui relève de la réalité à propos de ce statut «international».

47. Ce constat avait fait dire à un ancien consul américain au Canada, qui s'adressait au journaliste Gérald Leblanc : «Vous avez de bons projets et de bons leaders, mais aucune cohésion, aucune orientation commune" dans «Destination nulle part», La Presse, 11 septembre 1989, p. A-5, une réalité que les membres de l'OCDE auraient baptisée du vocable évocateur de «Syndrome canadien».

48. Nous incluons dans ce groupe les administrateurs de la ville de Montréal et de la Communauté urbaine de Montréal.

49. C'est d'ailleurs l'idée que défend Benjamin Higgins dans son ouvrage sur Montreal : The Rise and Fall of Montreal?, Moncton, The Canadian Institute for Research on Regional Developpement, 1990. 


\section{Conclusion}

Les tendances qui se dégagent de l'analyse des données recueillies en entrevue et de la recherche documentaire nous permettent d'identifier certains mécanismes et le cadre dans lequel veulent dorénavant travailler les principaux acteurs. On constate donc l'émergence d'une dynamique nouvelle entre les principaux acteurs montréalais qui ont mis de l'avant les moyens de réaliser leur politique. En effet, en mettant sur pied des mécanismes de concertation, ils s'assurent de présenter un minimum de cohésion face à leurs interlocuteurs, notamment les gouvernements supérieurs. On note également chez les acteurs un réel désir et une volonté d'exercer une prise en charge qui tranche avec l'attentisme trop marqué du passé. On observe en outre l'émergence, parmi ces mêmes acteurs, d'un nouveau leadership d'affaires qui envisage le développement économique dans une perspective de développement culturel et social. II est clair aussi que tous ces acteurs veulent absolument développer une vision à plus long terme. Enfin, ils ont pris conscience que c'est dans la "ville» que se joueront les principaux enjeux de demain.

Ces nouvelles tendances sont de bon augure mais doivent se conjuguer avec l'ouverture de créneaux spécifiques aptes à différencier Montréal de ses rivales. II faudrait donc que la métropole "se donne une vocation" (Laurent Picard), «se type" (Jean-Paul L'Allier) et "se différencie" (Daniel Latouche). Celui-ci précisait ainsi sa pensée :

Il est à parier qu'au tournant du millénaire, le nombre de musées, de troupes de danse et de théatre, la qualité du réseau des galeries et surtout l'ampleur des mégainstitutions culturelles seront des critères de localisation plus importants pour les multinationales, les notres y compris, que les infrastnuctures routières ou le taux de taxation ${ }^{50}$.

50. Daniel Latouche «La GI quoi? * Le Devoir, 22 décembre 1989, p. 9. 
En ce sens, une future politique de développement du rôle international des villes devra se baser sur une philosophie et une éthique différentes de celles qui inspirent actuellement nos administrations publiques. La dévolution des responsabilités au niveau local allant en augmentant, une nouvelle relation est en train de se développer entre l'État et les gouvernements locaux. À cet égard, le maire de Montpellier avait raison de dire :

La technopole, ce n'est rien d'autre que l'interface rapide entre l'intelligence, la recherche, l'innovation et la production. Dans l'Europe de 1992 ce seront d'abord des villes qui compteront. Quelles villes? Celles qui seront fortes, ouvertes et dynamiques, celles qui retrouveront la grande tradition des cités du Moyen Âge. Mon modèle, c'est la Florence de Médicis, dont le développement reposait sur l'alliance de la finance, de l'intelligence et de la culture et, bien sûr, le commerce ${ }^{51}$.

Par ailleurs, les acteurs présents et actifs dans le dossier de Montréal devront voir à intensifier la participation des syndicats, des groupes communautaires, des universités et de tous ceux qui auraient une contribution à apporter. Comme disait l'un de nos répondants : "Nous ne sommes pas assez nombreux pour nous balkaniser», et "le contexte féroce de la concurrence ne nous permet plus de nous payer le luxe de créer des empires".

Mentionnons en terminant que l'analyse de Jobert et Muller, qui nous a servi de cadre, débouchait sur I'hypothèse néo-corporatiste. Suivant cette hypothèse, l'émergence et la mise en œuvre des politiques publiques reposent en grande partie sur le modèle de relations que les politiciens et les partis entretiennent avec l'ensemble des groupes qui composent et structurent la médiation sociale. Ainsi, la majorité des lois ne

51. Olivier Peretié, «Montpellier toujours surdouée? ", Le Nouvel Observateur, $n^{\circ} 1264$, p. 8. 
dériveraient pas des programmes électoraux des partis politiques. Elles seraient plutôt l'initiative d'administrations particulièrement habiles à faire surgir les coopérations nécessaires, voire obligées, entre les principaux acteurs socioéconomiques. Pour ces auteurs, la concertation comme mode de gouvernement serait supérieure au parlementarisme, car elle seule peut rendre possibles les consensus nécessaires dans la conduite de l'action publique. Les oppositions entre les différents groupes de pression ne feraient que diviser les partis politiques. Les acteurs institutionnels que nous avons rencontrés partagent de plus en plus ce point de vue. II reste à voir comment ce discours s'actualisera dans les années à venir. 
TABLEAU I

Elémenté d'une politique d'internationalisation de la Ville de Montréal '

\begin{tabular}{|c|c|c|c|c|}
\hline $\begin{array}{l}\text { MESURES VISANT } \\
\text { LA CONCERTA. } \\
\text { TION }\end{array}$ & $\begin{array}{l}\text { MESURES D'AIDE } \\
\text { AUX INDUSTRIES } \\
\text { AU COMMERCE ET } \\
\text { AUX INVESTISSE- } \\
\text { MENTS }\end{array}$ & MESURES DE SOUTIEN & $\begin{array}{l}\text { MESURES VISANT LA } \\
\text { CREATION DE RE- } \\
\text { SEAUX }\end{array}$ & $\begin{array}{l}\text { MESURES VISANT } \\
\text { A ATTIRER DES } \\
\text { ONGIOING ET DES } \\
\text { EVENEMENTS } \\
\text { INTERNATIONAUX }\end{array}$ \\
\hline $\begin{array}{l}\text { - Mesures d'aide } \\
\text { visant à faciliter, à } \\
\text { générer un climat de } \\
\text { concertation: 1) entre } \\
\text { les élus; 2) entre les } \\
\text { différents partenaires. } \\
\text { - Mesures d'aide au } \\
\text { recrutement, à la } \\
\text { promotion d'edminis- } \\
\text { trateurs et de profes- } \\
\text { sionnels habilités à } \\
\text { gérer selon le modèle } \\
\text { très exigeant de la } \\
\text { concertation. } \\
\text { - Mesures d'aide à la } \\
\text { concertation interuni- } \\
\text { versitaire (ex. pour ré- } \\
\text { pondre à certains be- } \\
\text { soins comme la pé- } \\
\text { nurie de chercheurs } \\
\text { dans certains domai- } \\
\text { nes). }\end{array}$ & $\begin{array}{l}\text { - Mesures d'aide aux } \\
\text { investisseurs étran- } \\
\text { gers et mesures de } \\
\text { repérage des inves- } \\
\text { tissements étrangers } \\
\text { (ex. Corporation exis- } \\
\text { tant à cette fin dans } \\
\text { la ville de Boston). } \\
\text { - Mesures d'aide pour } \\
\text { attirer les sièges so- } \\
\text { ciaux et les multina- } \\
\text { tionales (ex. les dis- } \\
\text { positions fiscales). } \\
\text { - Mesures d'aide au } \\
\text { développement de } \\
\text { cróneaux industriels } \\
\text { pour favoriser la com- } \\
\text { pétence et par con- } \\
\text { séquent la concur- } \\
\text { rence dans certains } \\
\text { domaines comme la } \\
\text { technologie, le de- }\end{array}$ & $\begin{array}{l}\text { Mesures d'aide au } \\
\text { développement et à l'amé- } \\
\text { lioration des transports } \\
\text { (aérien, portuaires, rou- } \\
\text { tiers, ferroviaires). } \\
\text { - Mesures d'aide à la } \\
\text { création d'une cité inter- } \\
\text { nationale. } \\
\text { Mesures d'aide pour } \\
\text { informer les étrangers de } \\
\text { la situation particulière du } \\
\text { Québec en matière de } \\
\text { fiscalité, de législation du } \\
\text { travail ot de législation } \\
\text { linguistique. } \\
\text { - Mesures visant la créa- } \\
\text { tion d'un centre d'infor- } \\
\text { mation sur les questions } \\
\text { internationales (à l'instar } \\
\text { du centre mais sur pied à } \\
\text { Lille on France). } \\
\text { - Mesures d'aide à l'ob- } \\
\text { tention des crédits fédé- } \\
\text { raux disponibles (ex. à } \\
\text { l'ACDl et à la SEE) et à la } \\
\text { participation au proces- }\end{array}$ & $\begin{array}{l}\text { - Mesures visant à tirer } \\
\text { profit de la présence } \\
\text { d'étudiants étrangers au } \\
\text { sein de nos universités } \\
\text { (ex. Fédération des Étu- } \\
\text { diants d'Afrique Noire en } \\
\text { France). } \\
\text { - Mesures visant la créa- } \\
\text { tion d'une banque de } \\
\text { personnes-ressources } \\
\text { d'origine étrangère pré- } \\
\text { sentes sur le territoire et } \\
\text { susceptibles de devenir } \\
\text { des alliés du Québec dans } \\
\text { le monde (étudiants étran- } \\
\text { gers, immigrants ayant } \\
\text { gardé des contacts avec } \\
\text { leur pays d'origine) et } \\
\text { création d'une banque de } \\
\text { personnes-ressources } \\
\text { québécoises oeuvrant } \\
\text { dans les organismes in- } \\
\text { ternationaux, les ambas- } \\
\text { sades canadiennes ou les }\end{array}$ & $\begin{array}{l}\text { - Mesures visant à } \\
\text { attirer des ONG et } \\
\text { des OING et à main- } \\
\text { tenir de bonnes re- } \\
\text { lations avec les } \\
\text { organismes interna- } \\
\text { tionaux présents sur } \\
\text { notre territoire. } \\
\text { - Mesures d'aide } \\
\text { aux grands óvéne- } \\
\text { ments internatio- } \\
\text { naux existants ou à } \\
\text { créer (scientifiques, } \\
\text { sportifs, culturels, } \\
\text { touristiques, etc.). } \\
\text { - Mise sur pied d'un } \\
\text { centre de données } \\
\text { informatisées sur la } \\
\text { coopératione. } \\
\text { 6. Cette idbe eat tirbe } \\
\text { d'une communication faite } \\
\text { per Mme Gabrielle Le- }\end{array}$ \\
\hline
\end{tabular}
concernis. 


\begin{tabular}{|c|c|c|c|c|}
\hline $\begin{array}{l}\text { Mesures visant la } \\
\text { coopération inter- } \\
\text { services etatiques } \\
\text { provinciaux et inter- } \\
\text { services entre les } \\
\text { organisations publi- } \\
\text { ques et prives à visée } \\
\text { internationale ou } \\
\text { ayant des incidences } \\
\text { internationales (ex. Le } \\
\text { Conseil international } \\
\text { de coordination de } \\
\text { Boston). } \\
\text { - Mesures d'aide à la } \\
\text { concertation et au dé- } \\
\text { veloppement des } 350 \\
\text { unités privées de re- } \\
\text { cherche industrielle } \\
\text { avec la dizaine de cen- } \\
\text { tres de recherche pu- } \\
\text { blics. }\end{array}$ & $\begin{array}{l}\text { sign, la culture, les } \\
\text { communications, les } \\
\text { équipements de télé- } \\
\text { communications, le } \\
\text { matériel de transport, } \\
\text { l'aéronautique, l'éner- } \\
\text { gie, les produits phar- } \\
\text { maceutiques, les ma- } \\
\text { tériaux et les procé- } \\
\text { dés industriels, le tou- } \\
\text { risme et la restaura- } \\
\text { tion. } \\
\text { - Mesures d'aide à la } \\
\text { recherche et au déve- } \\
\text { loppement. } \\
\text { - Mesures d'aide au } \\
\text { commerce internatio- } \\
\text { nal (ex. amélioration } \\
\text { de l'expertise, de la } \\
\text { formation des profes- } \\
\text { sionnels oeuvrant dans } \\
\text { ce secteur). }\end{array}$ & 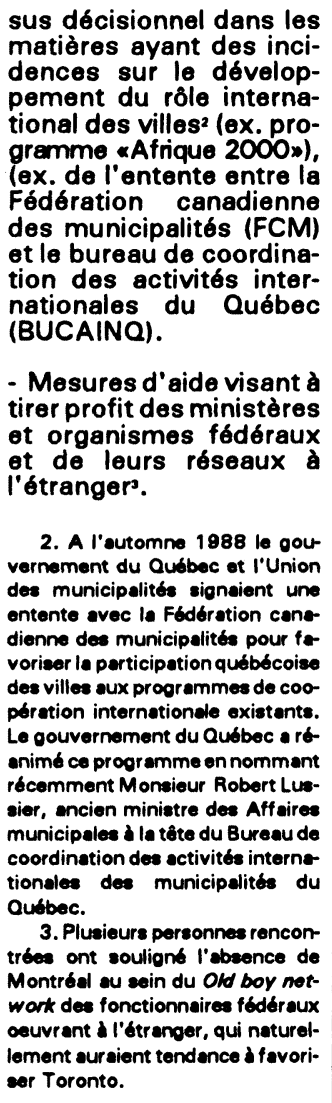 & 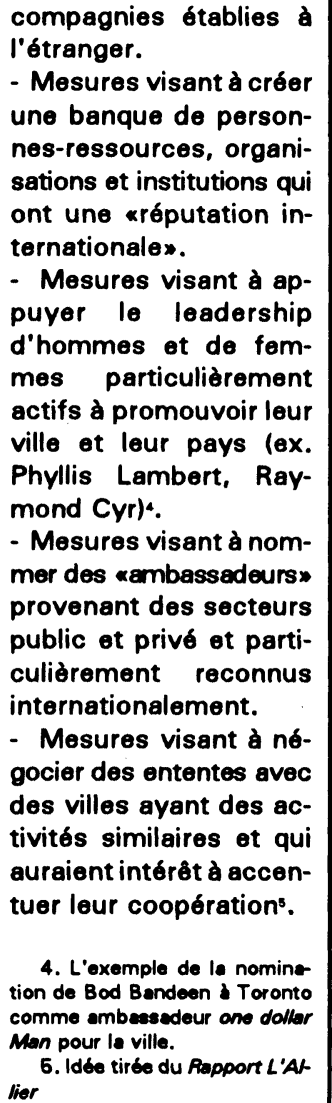 & 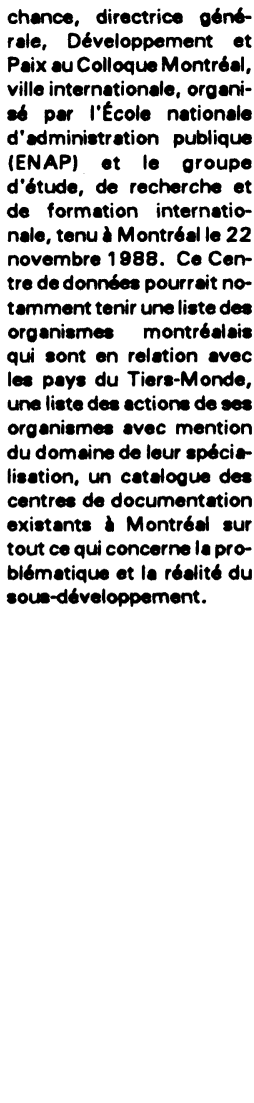 \\
\hline
\end{tabular}

